\title{
Nonequilibrium Probing of Two-Level Charge Fluctuators Using the Step Response of a Single-Electron Transistor
}

\author{
A. Pourkabirian, ${ }^{1}$ M. V. Gustafsson, ${ }^{1}$ G. Johansson, ${ }^{1}$ J. Clarke, ${ }^{1,2}$ and P. Delsing ${ }^{1}$ \\ ${ }^{1}$ Microtechnology and Nanoscience, Chalmers University of Technology, S-41296 Göteborg, Sweden \\ ${ }^{2}$ Department of Physics, University of California, Berkeley, California 94720, USA
}

(Received 27 August 2014; published 19 December 2014)

\begin{abstract}
We report a new method to study two-level fluctuators (TLFs) by measuring the offset charge induced after applying a sudden step voltage to the gate electrode of a single-electron transistor. The offset charge is measured for more than $20 \mathrm{~h}$ for samples made on three different substrates. We find that the offset charge drift follows a logarithmic increase over 4 orders of magnitude in time and that the logarithmic slope increases linearly with the step voltage. The charge drift is independent of temperature, ruling out thermally activated TLFs and demonstrating that the charge fluctuations involve tunneling. These observations are in agreement with expectations for an ensemble of TLFs driven out of equilibrium. From our model, we extract the density of TLFs assuming either a volume density or a surface density.
\end{abstract}

DOI: 10.1103/PhysRevLett.113.256801

PACS numbers: 73.20.Hb, 05.40.Ca, 85.35.Gv

Two-level fluctuators (TLFs) are found in many, if not all, solid-state systems. The microscopic origin and physics of TLFs have been extensively studied in mesoscopic physics over the last 3 decades [1-7]. It is generally believed that an ensemble of TLFs gives rise to charge noise with a $1 / f$-like power spectrum that limits the performance of all charge sensitive devices [8,9], including single-electron transistors (SETs) [10], quantum point contacts [11] and quantum capacitance detectors [12]. TLFs also induce decoherence in solid-state qubits that are the building blocks in quantum information processing [13-15]. Although there are various models for TLFs [16-19], their physical origin and location remain open questions. The simplest microscopic model consists of a two-well potential containing a charged particle. Depending on the height and width of the barrier separating the wells and on temperature, the particle is transferred from one well to the other either by thermally activated hopping [20,21] or by tunneling [22]. There are also different scenarios regarding the location of the TLFs: they may be distributed in the volume of the substrate (volume distribution) [23] or at the interfaces between metals and insulators (surface distribution). Earlier, we suggested [17] that charge noise may arise from electrons tunneling back and forth between the Fermi sea in the metallic electrode and traps at the metal-insulator interface such as localized metal-induced gap states (MIGs); MIGs have been proposed as a possible origin of the localized magnetic moments giving rise to flux noise in superconducting quantum interference devices and flux qubits [24].

SETs are used as electrometers to study TLFs. The SET [Fig. 1(a)] is extremely sensitive to charge [25,26]. When the SET is voltage biased, the current, $I_{\mathrm{SET}}$, is periodically modulated by the charge induced on its island by a nearby gate [Fig. 1(a)] with period $e / C_{g}$. Here, $C_{g}$ is the capacitance between the island and the gate electrode, and $e$ is the electron charge. Consequently, a fluctuating charge in the vicinity of the SET causes $I_{\mathrm{SET}}$ to fluctuate.

In virtually all previous work on TLFs and charge noise, the ensemble of TLFs is close to equilibrium and the data acquired in these experiments have generally not conclusively shown whether the mechanism is thermal activation or tunneling.

In this Letter, we take another approach and investigate the response of TLFs driven far out of equilibrium by a strong external electric field. We present measurements of the charge drift $Q$ following the application of a sudden step voltage $\Delta V$ to the SET gate, which causes the induced charge on the SET island to increase rapidly. Remarkably, the charge drifts slowly long after the step is applied

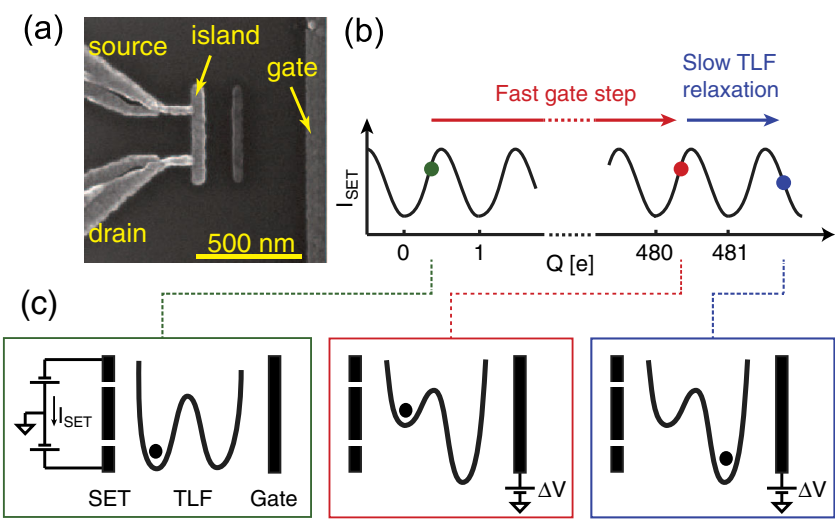

FIG. 1 (color online). (a) Micrograph of a SET with typical junction size of $20 \times 50 \mathrm{~nm}^{2}$. [(b), (c)] Schematic overview of the measurements. Starting from equilibrium (green), we apply a sudden voltage step $\Delta V$ to the gate, bringing the TLF ensemble out of equilibrium (red). After the step, we use the SET to record the charge drift $Q(t)$ as the TLFs relax to their new ground states (blue). 
[Fig. 1(b)]. We argue that this drift is due to the change in the potential landscapes of the TLFs caused by the gate voltage. Some of the TLFs are brought to metastable states, which decay after a characteristic time causing the charge drift [Fig. 1(c)]. We have measured this drift for up to $20 \mathrm{~h}$ for several SETs made on three different kinds of substrates. We find that the drift increases logarithmically with time and is independent of temperature, allowing us to rule out thermally activated TLFs. Furthermore, we have measured the response to voltage steps with different heights and found that the logarithmic slope of the drift increases with increasing voltage. We show that this behavior is consistent with the response of an ensemble of TLFs, and we develop a theory from which we can extract the densities for these TLFs.

We made measurements on samples with nominally identical layouts fabricated on three different substrates: glass, sapphire, and oxidized silicon with an oxide thickness of $400 \mathrm{~nm}$. The aluminum SETs were fabricated with electron-beam lithography and double-angle evaporation [27]. All measurements were performed in a dilution refrigerator with a base temperature of $20 \mathrm{mK}$. A magnetic field of $1 \mathrm{~T}$ quenched superconductivity in the aluminium. We present results for four representative samples, with extracted parameters shown in Table I.

In all experiments, the SET was biased symmetrically with respect to ground and we stepped the gate voltage abruptly while sampling $I_{\text {SET }}$ continuously at $2 \mathrm{k}$ samples/s. Figure 2(a) shows $I_{\mathrm{SET}}(t)$ for device 1 at a temperature $T=30 \mathrm{mK}$. The sample was left for a long time at a gate voltage $V_{g}=-4.9 \mathrm{~V}$ and $V_{g}$ then stepped to $+4.9 \mathrm{~V}$, giving a step height of $\Delta V=9.8 \mathrm{~V}$. Figure 2(b) shows the charge induced on the island extracted from the data in Fig. 2(a) by counting the number of oscillations in $I_{\mathrm{SET}}$, each one of which corresponds to an additional electron induced on the island. The inset in Fig. 2(b) shows the same data on a logarithmic scale. We see that the offset charge increases logarithmically over more than 4 decades of time.

We note that we cannot count the total number of electrons induced on the island since hundreds of electrons are induced instantaneously when we apply the voltage step. We can, however, estimate the initial change from $C_{q}$. For device 1, the initial charge jump was $\sim 480 e$. Thus, the additional slow drift of about $10 e$ follows the initial step of $480 e$ [Fig. 1(b)].
To obtain a direct measurement of the charge drift, we used a proportional-integral-derivative (PID) regulator in subsequent experiments, feeding the regulation signal to the gate to maintain a constant SET current. Since the regulation commenced $\sim 1 \mathrm{~s}$ after the voltage step was applied, we cannot capture the first few seconds of the drift. Figure 3 shows the measured charge drift using both methods for different devices for almost $20 \mathrm{~h}$ after applying the voltage step. From the data in Fig. 3, we extract the logarithmic slopes of the charge drift normalized to the step height,

$$
H=\frac{1}{\Delta V} \frac{\Delta Q(t)}{\Delta \log t},
$$

summarized in Table I. Comparing the two measurements for device 1 at $30 \mathrm{mK}$ and $2 \mathrm{~K}$, we see that $H$ does not depend significantly on $T$ in this range.

To investigate the dependence of the total measured drift on $\Delta V$, in separate measurements we applied voltage steps with different heights to device 3 and measured the charge drift. Figure 4 shows that the rate of charge drift is proportional to $\Delta V$.

The simplest microscopic model for a TLF is a charged particle trapped in a double-well potential with an energy difference $\Delta E=E_{R}-E_{L}$ between the right and the left well and with an energy barrier of height $E_{b}$ [Figs. 5(a), 5(b)]. The charge, which we assume to be the electron charge $e$, moves a distance $d$ between the two locations either by thermal activation over the barrier [Fig. 5(a)] or by tunneling through the barrier [Fig. 5(b)]. The motion is characterized by a switching time $\tau=1 / \omega_{0}$, where $\omega_{0}$ is the sum of the forward and backward rates that depend on the properties of the TLF potential and on $T$. The equilibrium population of the right well is given by the Fermi distribution $f(\Delta E)$. An alternative model is a charge that moves between the Fermi gas in one of the electrodes and a well with energy $\Delta E$ compared to the Fermi energy and energy barrier $E_{b}$. For the purpose of this work, these two models behave in the same way, but we base our description on the double-well TLF.

To illustrate how the TLFs influence the SET in our experiment, we consider a simple parallel-plate capacitor model, where one plate consists of the gate and the other one of the SET island and leads [28]. Figure 5(c) shows

TABLE I. Extracted parameters for the four measured SETs.

\begin{tabular}{lccccc}
\hline \hline Device & Substrate & $\begin{array}{c}E_{C} / k_{B}{ }^{\mathrm{a}} \\
(\mathrm{K})\end{array}$ & $C_{g}(\mathrm{aF})$ & $T(\mathrm{mK})$ & $\begin{array}{c}H=(1 / \Delta V)(\Delta Q(t) / \Delta \log t) \\
{[e /(\mathrm{V} \cdot \text { decade of } t)]}\end{array}$ \\
\hline 1 & $\mathrm{Si}-S i O_{X}$ & 4.1 & 7.8 & 2000 & 0.26 \\
1 & ${\mathrm{Si}-\mathrm{SiO}_{X}}$ & 4.1 & 7.8 & 30 & 0.22 \\
2 & ${\mathrm{Si}-\mathrm{SiO}_{X}}_{3}$ & 5.8 & 10 & 20 & 0.22 \\
4 & $\mathrm{Glass}$ & 4.2 & 9.2 & 20 & 0.37 \\
\hline \hline
\end{tabular}

${ }^{\mathrm{a}} E_{C}$ is the charging energy of the SET, the energy required to charge the SET island with one electron. 

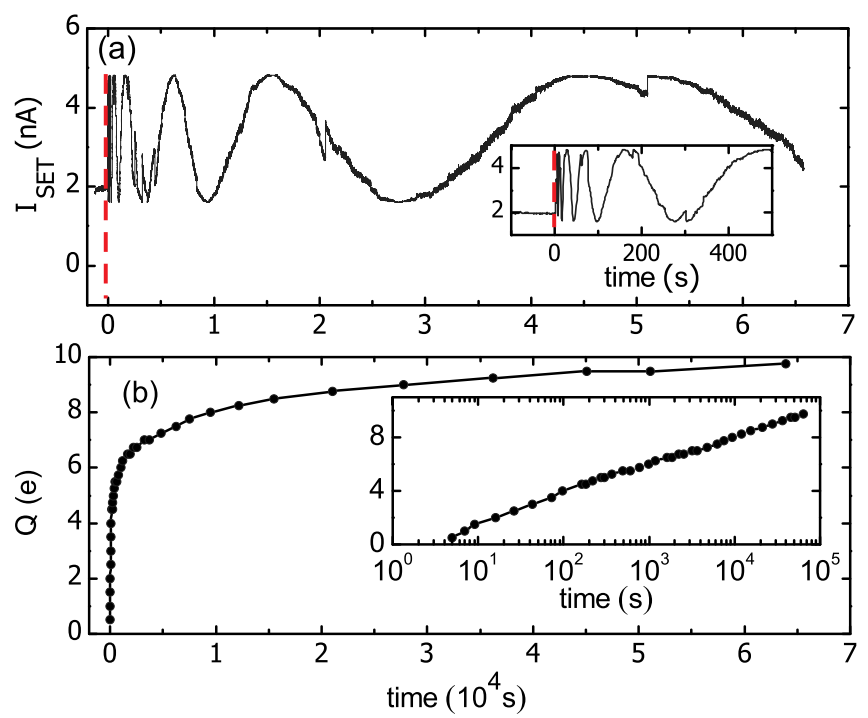

FIG. 2 (color online). Step response measurements at $T=$ $30 \mathrm{mK}$ on device 1. (a) Continuous measurement of $I_{\text {SET }}$ over a period of about $20 \mathrm{~h}$. A step voltage $\Delta V=9.8 \mathrm{~V}$ was applied to the gate at $t=0$ (red dashed lines). The inset shows the first $500 \mathrm{~s}$ after the step. (b) Charge drift extracted from (a). The inset shows the same data on a logarithmic time axis; the charge increases logarithmically with time.

such a geometry. Each TLF has a different angle $\theta$ between its displacement vector $\vec{d}$ and the gate field lines $\vec{E}_{G}(\vec{r})$ [solid lines in Fig. 5(c)].

First, we consider the effect on the TLFs of a voltage step. The electric field changes the energy difference between the two wells by $\delta E=e \Delta V \vec{d} \cdot \vec{e}_{G}(\vec{r})$, where we have defined the normalized gate field $\vec{e}_{G}(\vec{r})=\vec{E}_{G}(\vec{r}) / \Delta V$. To determine how much charge a TLF in a given location induces on the SET island, we calculate the electric field $\vec{E}_{V}(\vec{r})$ in a virtual situation, where the island is held at a potential $V_{0}$ and the other electrodes are grounded [dashed lines in Fig. 5(c)]. We define the normalized virtual field as $\vec{e}_{V}(\vec{r})=\vec{E}_{V}(\vec{r}) / V_{0}$. For a TLF at any point $\vec{r}$ in space, the change in charge induced on the island by a switching event is given by $\delta q(\vec{r})=e \vec{d} \cdot \vec{e}_{V}(\vec{r})[29,30]$.

The equilibrium population of each TLF is determined by its energy difference $\Delta E$, which changes by an amount $\delta E$ as the gate voltage step is applied [Figs. 5(a), 5(b)]. The charge distribution approaches the new equilibrium on a time scale set by the (new) switching time $\tau^{\prime}$. We assume an ensemble of TLFs with different final switching times $\tau^{\prime}$, different initial energy differences $\Delta E$, and different displacement vectors $\vec{d}$ and sum the contribution from a set of $N$ TLFs to find the total charge induced on the island

$Q(t)=\sum_{i=1}^{N} \delta q_{i}\left[f\left(\Delta E_{i}\right)-f\left(\Delta E_{i}-\delta E_{i}\right)\right]\left(1-e^{-t / \tau_{i}^{\prime}}\right)$,

where the subscript $i$ refers to the individual TLF values.

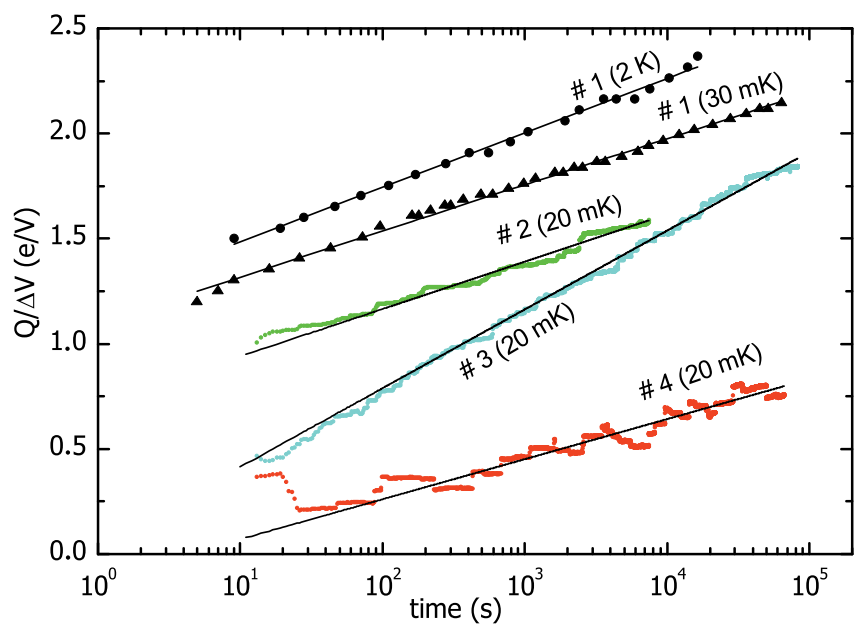

FIG. 3 (color online). Normalized charge drift measured for four different devices. The curves have been offset vertically for clarity. For device 1, the charge drift was extracted from the current modulation of the SET. For devices 2-4, a PID loop was connected to the gate (see text). The measurements on different SETs on different substrates show a logarithmic charge drift with similar slopes. The charge drift per decade of time in Table I is extracted from a least-squares fit to each trace (solid black lines).

We now assume (i) that the TLFs are numerous enough to change the sum into an integral, (ii) a flat distribution of initial energy differences, and (iii) that the logarithm of the switching times $\log (\tau)$ has a flat distribution between minimum and maximum switching times $\tau_{\min }=1 / \omega_{\max }$ and $\tau_{\max }=1 / \omega_{\min }$. These assumptions lead to the observed $1 / f$ power spectrum for the noise [1] and would arise naturally from a roughly flat distribution of barrier heights in the case of thermal activation and barrier widths in the case of quantum tunneling when the barrier height is the

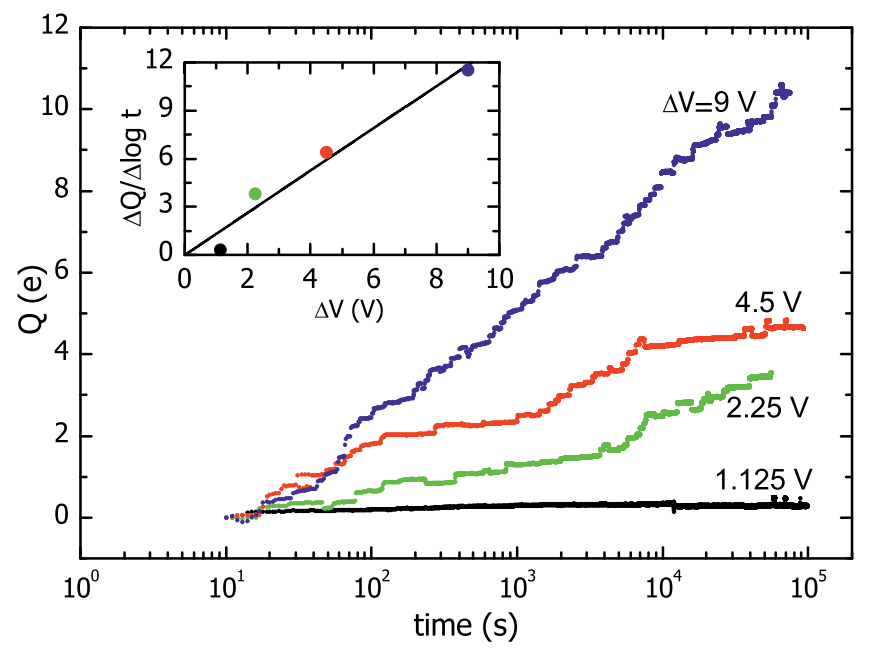

FIG. 4 (color online). Charge drift for different voltage step heights $\Delta V$ for device 3 at $20 \mathrm{mK}$. The logarithmic slope of the charge drift $\Delta Q(t) / \Delta \log t$ increases with $\Delta V$. The inset shows $\Delta Q(t) / \Delta \log t$ vs $\Delta V$, and the line represents a least-squares fit constrained to pass through the origin. 
(a)

\section{before the step}

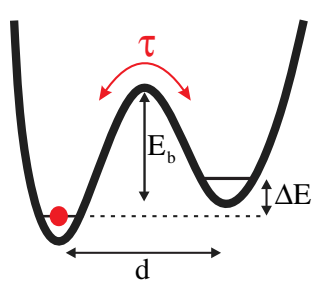

(b)

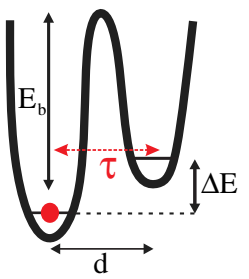

after the step

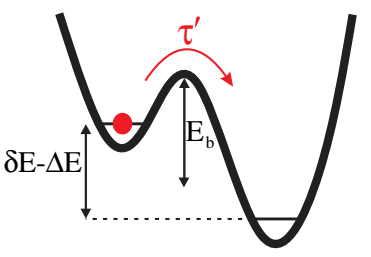

$\delta \mathrm{E}-\Delta \mathrm{E}$

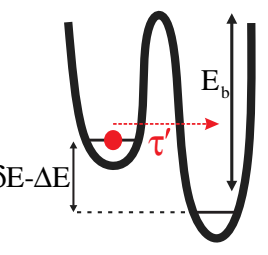

(c)

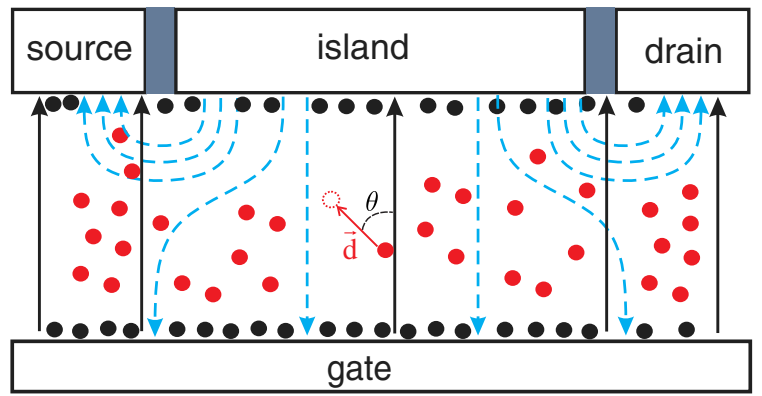

FIG. 5 (color online). Microscopic model of a TLF and its influence on the SET. [(a),(b)] A charged particle in a double potential well with a distance $d$ between the two wells, barrier height $E_{b}$, and energy difference $\Delta E$ between the two states. Left: before applying the step, the switching time is $\tau$. Right: after applying the step, the energy difference between the two wells changes by $\delta E$, and the switching time changes to $\tau^{\prime}$. $E_{b}$ is defined with respect to the mean between the two states and does not change to first order. (a) Thermal activation. (b) Quantum tunneling. (c) Simplified geometry of the SET, gate, and TLFs. At the center, an individual TLF is shown schematically with an angle $\theta$ between its displacement vector $\vec{d}$ and the gate field $\vec{E}_{G}(\vec{r})$ (solid lines). The dashed lines show the virtual field, $\vec{E}_{V}(\vec{r})$ (see text). The red and black circles represent the volume and surface distributions of TLFs, respectively.

largest energy of the system. For $\tau_{\min } \ll t \ll \tau_{\max }$ we find (see the Supplemental Material [31])

$Q(t) \approx \frac{\ln \left(t / \tau_{\min }\right)+\gamma}{\ln (10)} \int n(\vec{r}, \theta) \delta q(\vec{r}, \theta) \delta E(\vec{r}, \theta) d \vec{r} d \theta$,

where $\gamma$ is Euler's gamma and $n(\vec{r}, \theta)$ is the density of TLFs with displacement vector oriented along the direction $\theta$, per unit energy difference and per decade of switching time. The $\ln (10)$ term in the denominator of Eq. (3) stems from our definition of the TLF density in units of TLFs per decade of frequency.

In the case of quantum tunneling the switching rates are independent of temperature, and the effect of temperature is basically to broaden the population according to the Fermi distribution. This has no effect on the step response.
In the case of thermal activation, the switching rate is determined by $T, E_{b}$, and the attempt frequency $\Omega$ according to $\omega_{0}=\Omega e^{-E_{b} / k_{B} T}$. If we consider $\Omega$ and $E_{b}$ to be independent of $T$ and that $E_{b}$ has a flat distribution, the TLF density per decade scales linearly with $T$. It follows from Eq. (3) that the step response should then also be proportional to $T$ (Supplemental Material [31]). In our experiments $\delta E \gg k_{B} T$, and therefore, the voltage step actually flattens out the TLFs that are thermally active at equilibrium, since $E_{b} \sim k_{B} T$. These TLFs will, thus, switch immediately, and only those with a remaining barrier height comparable to $k_{B} T$ will contribute to the slow charge drift. Assuming a flat distribution of final barrier heights, the slow charge drift would be independent of $\Delta V$.

To compare with experiment, we calculate the parameter $H$ [Eq. (1)]. We consider two special cases: homogeneous volume and homogeneous surface distributions of $n(\vec{r})$. In the case of a homogeneous volume distribution $n_{v}$, we assume that the TLF can be randomly oriented. We obtain (Supplemental Material [31])

$$
H_{v}=\frac{e^{2} d^{2} n_{v}}{3} \int \vec{e}_{V}(\vec{r}) \cdot \vec{e}_{G}(\vec{r}) d V .
$$

For a homogeneous surface distribution $n_{s}$, we assume that the electrons tunnel perpendicularly from the metal surface $S$ to the trap, i.e., $\theta=0$. We obtain

$$
H_{s}=e^{2} d^{2} n_{s} \int \vec{e}_{V}(\vec{r}) \cdot \vec{e}_{G}(\vec{r}) d S .
$$

It is interesting to note that the change in charge can be either positive or negative. In particular, when a voltage is applied to the gate, a TLF situated directly underneath the SET island and a TLF underneath the drain or source will both switch in the same direction. However, since $\vec{e}_{V}(\vec{r})$ points in opposite directions at the two locations, the induced charge from the TLFs underneath the drain and source will have the opposite sign compared to the contribution from the TLFs underneath the SET island (Supplemental Material [31]). Thus, the sign of a particular charge change (see e.g., device 4 in Fig. 3) provides information about the location of an individual TLF.

Our experimental data clearly show that $Q$ increases logarithmically with time after the voltage step. Although charge drift with a similar behavior has been reported previously [32-34], the dependence on time was not analyzed. We note also that logarithmic time dependencies are well known in glassy systems [35]. For example, Vaknin et al. [36] found a $\ln (t)$ dependence of the excess electrical conductance in thin films of amorphous $\mathrm{InO}_{x}$ following a sudden application of a voltage to a nearby gate and ascribed this behavior to variable-range hopping using a model related to ours. At liquid helium temperatures, the temperature dependence of the conductance of similar samples depended strongly on the carrier concentration [37]. We are intrigued that systems involving such different physics should also exhibit a $\ln (t)$ dependence. 
Studying the details of this charge drift, we draw a number of conclusions about the TLFs. (i) The charge drift appears not to depend on temperature (Table I). This indicates that the charge transfer mechanism is tunneling and not thermal activation. (ii) We find that the charge drift increases approximately linearly with $\Delta V$ (Fig. 4), indicating that the distribution of $\Delta E$ for the TLFs is uniform. This also speaks against thermal activation, since in that case, the drift would not depend on $\Delta V$ (Supplemental Material [31]). Furthermore, tunneling is consistent with our previous measurements of linear temperature dependence of the charge noise spectral density [17]. (iii) The charge drift is similar for devices fabricated on different materials. (iv) Using the model described earlier and the measured values for $H$, we extract the density of TLFs from Eqs. (4) and (5) by calculating the integrals numerically for the actual geometry (Supplemental Material [31]). Assuming $d \approx 1 \mathrm{~nm}$, we estimate the densities to be $n_{v} \approx 1.5 \times 10^{24}$ $\left(\mathrm{m}^{3} \mathrm{eV} \text { decade }\right)^{-1}$ and $n_{s} \approx 1.6 \times 10^{16}\left(\mathrm{~m}^{2} \mathrm{eV} \text { decade }\right)^{-1}$, for the volume and surface cases, respectively. The extracted surface density is similar to the density predicted for MIGs [24].

The work was supported by the Swedish Research council and by the Knut and Alice Wallenberg Foundation. We thank SangKook Choi, Jared Cole, Steven Louie, Samira Nik, Eva Olsson, and Lunjie Zeng for very helpful discussions. J. C. thanks the faculty of Chalmers University for their hospitality during his tenure of the Chalmers 150th Anniversary Visiting Professorship during which some of the experiments were performed. His research was funded by the Office of the Director of National Intelligence (ODNI), Intelligence Advanced Research Projects Activity (IARPA), through the Army Research Office.

[1] E. Paladino, Y. Galperin, G. Falci, and B. Altshuler, Rev. Mod. Phys. 86, 361 (2014).

[2] L. R. Simkins, D. G. Rees, P. H. Glasson, V. Antonov, E. Collin, P. G. Frayne, P. J. Meeson, and M. J. Lea, J. Appl. Phys. 106, 124502 (2009).

[3] D. G. Rees, P. Glasson, L. R. Simkins, E. Collin, V. Antonov, P. G. Frayne, P. J. Meeson, and M. J. Lea, Appl. Phys. Lett. 93, 173508 (2008).

[4] M. Kenyon, J. L. Cobb, A. Amar, D. Song, N. M. Zimmerman, C. Lobb, and F. C. Wellstood, IEEE Trans. Appl. Supercond. 9, 4261 (1999).

[5] M. Furlan and S. V. Lotkhov, Phys. Rev. B 67, 205313 (2003).

[6] N. M. Zimmerman, J. L. Cobb, and A. F. Clark, Phys. Rev. B 56, 7675 (1997).

[7] N. M. Zimmerman, W. H. Huber, B. Simonds, E. Hourdakis, A. Fujiwara, Y. Ono, Y. Takahashi, H. Inokawa, M. Furlan, and M. W. Keller, J. Appl. Phys. 104, 033710 (2008).

[8] C. T. Rogers and R. A. Buhrman, Phys. Rev. Lett. 53, 1272 (1984).

[9] S. Machlup, J. Appl. Phys. 25, 341 (1954).

[10] K. Likharev, IEEE Trans. Magn. 23, 1142 (1987).
[11] H. van Houten and C. Beenakker, Phys. Today 49, No. 7, 22 (1996).

[12] M. D. Shaw, J. Bueno, P. Day, C. M. Bradford, and P. M. Echternach, Phys. Rev. B 79, 144511 (2009).

[13] J. Clarke and F. K. Wilhelm, Nature (London) 453, 1031 (2008).

[14] J. Bylander, S. Gustavsson, F. Yan, F. Yoshihara, K. Harrabi, G. Fitch, D. G. Cory, Y. Nakamura, J.-S. Tsai, and W. D. Oliver, Nat. Phys. 7, 565 (2011).

[15] Y. Makhlin, G. Schön, and A. Shnirman, Rev. Mod. Phys. 73, 357 (2001).

[16] S. Kafanov, H. Brenning, T. Duty, and P. Delsing, Phys. Rev. B 78, 125411 (2008).

[17] M. V. Gustafsson, A. Pourkabirian, G. Johansson, J. Clarke, and P. Delsing, Phys. Rev. B 88, 245410 (2013).

[18] T. C. DuBois, M. C. Per, S. P. Russo, and J. H. Cole, Phys. Rev. Lett. 110, 077002 (2013).

[19] M. Kenyon, C. J. Lobb, and F. C. Wellstood, J. Appl. Phys. 88, 6536 (2000).

[20] P. Dutta and P. Horn, Rev. Mod. Phys. 53, 497 (1981).

[21] O. Astafiev, Y. A. Pashkin, Y. Nakamura, T. Yamamoto, and J. S. Tsai, Phys. Rev. Lett. 96, 137001 (2006).

[22] Z. Kim, V. Zaretskey, Y. Yoon, J. F. Schneiderman, M. D. Shaw, P. M. Echternach, F. C. Wellstood, and B. S. Palmer, Phys. Rev. B 78, 144506 (2008).

[23] A. B. Zorin, F.-J. Ahlers, J. Niemeyer, T. Weimann, H. Wolf, V. A. Krupenin, and S. V. Lotkhov, Phys. Rev. B 53, 13682 (1996).

[24] S. K. Choi, D.-H. Lee, S. G. Louie, and J. Clarke, Phys. Rev. Lett. 103, 197001 (2009).

[25] A. Aassime, D. Gunnarsson, K. Bladh, P. Delsing, and R. J. Schoelkopf, Appl. Phys. Lett. 79, 4031 (2001).

[26] H. Brenning, S. Kafanov, T. Duty, S. Kubatkin, and P. Delsing, J. Appl. Phys. 100, 114321 (2006).

[27] G. J. Dolan, Appl. Phys. Lett. 31, 337 (1977).

[28] The calculations, however, are performed using the real geometry of the device.

[29] W. R. Smythe, Static and Dynamic Electricity (McGraw-Hill, New York, 1939).

[30] E. Mathieson, Induced Charge Distributions in Proportional Detectors (Brookhaven National Laboratory, New York, 1939).

[31] See the Supplemental Material at http://link.aps.org/ supplemental/10.1103/PhysRevLett.113.256801 for derivation of the TLF densities and the details of the FEM calculations. It also contains a discussion section concerning temperature and voltage dependence of the step response.

[32] G. Zimmerli, R. L. Kautz, and J. M. Martinis, Appl. Phys. Lett. 61, 2616 (1992).

[33] A. Manninen, J. Kauranen, J. Pekola, A. Savin, M. Kamp, M. Emmerling, A. Forchel, M. Prunnila, and J. Ahopelto, Jpn. J. Appl. Phys. 40, 2013 (2001).

[34] A. Savin, A. Manninen, P. Kivinen, and J. Pekola, in Physics, Chemistry and Application of Nanostructures: Reviews and Short Notes to Nanomeeting 2001, edited by V.E. Borisenko, S. V. Gaponenko, and V. S. Gurin (World Scientific, Singapore, 2001), p. 485.

[35] A. Amir, Y. Oreg, and Y. Imry, Proc. Natl. Acad. Sci. U.S.A. 109, 1850 (2012).

[36] A. Vaknin, Z. Ovadyahu, and M. Pollak, Phys. Rev. B 61, 6692 (2000).

[37] Z. Ovadyahu, Phys. Rev. Lett. 99, 226603 (2007). 